\title{
Use the Word Evolution
}

\author{
John N. Thompson
}

Published online: 20 November 2007

(C) Springer Science + Business Media, LLC 2007

Examples of rapid evolution abound in the scientific literature and are regularly reported in the popular press, and yet, most nonscientists think that evolution is a glacially slow process that is almost impossible for humans to observe. This disconnection between reality and perception is our fault, and the solution to correcting that misperception is simple, at least with respect to microevolutionary change.

Part of the problem is that we often fail to use the word evolution even when we mean exactly that. Instead, we have developed a rich vocabulary of alternatives that take the place of the word evolution in much scientific and popular writing. These alternatives or euphemisms are so common that they seem natural to us. I have collected some of the more common ones and a few of the less common ones in Table 1.

There is nothing wrong with sometimes using these euphemisms as a substitute for the word evolution. We all use them. Their use, though, has become so pervasive that some entire articles now appear on evolution without even once using the word evolution itself. Several years ago I read a well-written, three-page article on the evolution of resistance in agricultural pests in a major science journal that used more than half a dozen different alternatives to the word evolution, but used the actual word evolution only once. Many of us, myself included, commonly use a similarly high proportion of alternatives for the word evolution in our conversations, lectures, and articles.

You and I know that phrases such as "emergence of disease resistance" or "overcame defenses" mean evolution,

\section{J. N. Thompson $(\square)$}

Department of Ecology and Evolutionary Biology,

University of California, Santa Cruz,

Santa Cruz, CA 95060, USA

e-mail: thompson@biology.ucsc.edu but nonscientists often do not. When I have asked nonscientists what they think happened when a pathogen species "overcame resistance" in a new crop variety or a new antibiotic, they often respond by saying that, well, the pathogens changed. When I have asked them if they think that means that the pathogens evolved, they often give me a puzzled look. We make the connection or translation, but nonscientists and students do not.

We could therefore have a large effect on society's perception of evolution simply by using the word evolution when we mean it. We should skip the euphemisms, or use them sparingly. Pests evolve resistance to new crop varieties, pathogens evolve resistance to new antibiotics, and the traits of introduced species evolve in their new environments. If we were to make this simple change in our teaching and in our science writing, the next generation of nonscientists would be more likely to view evolution as a common, ongoing, and inevitable process rather than something that is slow and difficult to see in action.

I do not think that the proliferation of alternatives for the word evolution comes from any direct attempt to avoid the use of that word in most popular science writing. Rather, these alternatives have become common for other reasons. In all languages, some alternative, lessdirect words or phrases become clichés. Nowadays, when one is writing about the evolution of resistance in pathogens, the most common cliché phrase has become "developed resistance." These two words have become, to use another cliché phrase, inextricably linked. Once a cliché is established it becomes, by definition, hard to displace.

Historically, some alternatives to the word evolution have become established because they contain old uses of the word. The word evolution has been in the English language at least since the 1700s. One of the pre-Darwinian uses of the word was for an idea called the development 
Table 1 Some alternatives for the word evolution found in the scientific literature and in popular science writing

\begin{tabular}{ll}
\hline Alternative & Example (from one or more articles) \\
\hline Accelerate & ...pest resistance...accelerated \\
Acquire & ...acquired new traits \\
Become & ...weeds are becoming resistant \\
Change & ...influenza virus...frequently changes... \\
Create & ...creates...new strains \\
Develop & ...developed resistance \\
Emerge & ...emergence of resistance \\
Grow & ...pathogens have grown resistant \\
Overcome & ...overcome their vulnerability \\
Sprout & ...resistant [forms]...have begun sprouting and \\
& $\quad$ spreading \\
\hline
\end{tabular}

hypothesis. Lamarck's view of evolution was that it occurred through the inheritance of acquired characteristics. Hence, the words "developed" and "acquired," when we mean evolved, are carry-overs from past uses.

Yet another reason that alternatives have developed for the word evolution when writing especially about rapid evolution is that some writers erroneously equate evolution with sustained directional selection over long periods of time. By that view, rapid genetic change in populations does not really constitute evolution because it is not clear whether those changes will be sustained over geologic time. After all, a small change in the average size of the beaks of Galapagos finches during this decade may be undone by equally rapid genetic change in the other direction in the next decade.

There is nothing in the word evolution or, more importantly, in our understanding of the evolutionary process to justify that view. Evolution is quite simply heritable change in the genetic structure of a population. There is nothing in this or any similar standard definition of evolution that requires that genetic change in the average size, shape, or any other trait be maintained for a hundred, a thousand, or a million years before it can truly be called evolution. Evolution is simply evolution, and it is an inevitable consequence of heritable variation in populations living in constantly changing environments. In fact, much of evolution does not involved sustained, long-term directional change in the average characteristics of organisms. It involves natural selection cleaving off individuals with highly divergent traits within populations and favoring slightly different sets of traits within populations in each generation amid ongoing environmental change.

These continual small evolutionary changes in populations result in those populations meandering back and forth in their average sizes, shapes, physiological tolerances, and behaviors over time. These ongoing small evolutionary changes are what keep species in the evolutionary game. The average appearance of a species may not change much over time but populations are continually evolving as they respond to slight changes in their environments. For much of their evolutionary history, species are like a boat tied loosely to a dock. The average position of the boat does not change much over time, but the actual position of the boat in the water is constantly changing in response to changing tides and weather. Without the ability to shift up and down and back and forth with changing water conditions, the boat would be swamped.

We are therefore surrounded by instances of ongoing evolution, and we should take every opportunity we can to use the word whenever it is appropriate. Evolution is a good, clear word and does not need euphemisms to make it more interesting. 